\title{
Análisis comparativo de la gestión educativa como agente de cambio e innovación ante los complejos ambientes educativos, caso de las organizaciones: Colegio Nuevo Mundo y Colegio Técnico Profesional Piedades Sur, San Ramón
}

\author{
Comparative analysis of educational management as an agent of change and innovation to the complex \\ educational environments, for organizations: Nuevo Mundo High School and Piedades Sur's Technical High \\ School, San Ramon
}

Recibido 09 mayo 2013 • Aceptado 29 mayo 2013 • Corregido 22 junio 2013

\author{
María Alejandra Barquero González' \\ Sistema Educativo Saint Clare, \\ San José, Costa Rica \\ maalbago@gmail.com
}

\author{
Mirieth Montero Matamoros ${ }^{2}$ \\ Colegio Nuevo Mundo, \\ San José, Costa Rica \\ mirieth.montero@gmail.com
}

\begin{abstract}
Resumen. Este artículo aborda el análisis comparativo de la gestión educativa como agente de cambio e innovación ante los complejos ambientes educativos en las organizaciones: Colegio Nuevo Mundo y Colegio Técnico Profesional de Piedades Sur de San Ramón. Para obtener los datos se aplicó un cuestionario a una muestra de profesores y estudiantes, así como a los directores de ambas instituciones. Además de aprovechar múltiples fuentes secundarias, para construir un contexto de interpretación que permitiera ubicar el caso particular de estos dos centros educativos, dentro de la realidad de la gestión educativa como agente de cambio e innovación. El principio que dirige este estudio radica en que en la actualidad es indispensable la implementación de técnicas y estrategias, en donde se requieren líderes activos, con orientación humana y claridad en que la gestión educativa debe estar dirigida no solo en la educación del presente sino a la del futuro.

Finalmente, con los resultados y conclusiones que se obtuvieron a través del análisis de los datos, se realizó una propuesta de proyecto llamado "Semana de integración: fomentando una cultura de
\end{abstract}

\footnotetext{
Licenciada en Ciencias de la Educación con énfasis en Administración de la Educación de la Universidad de Costa Rica (UCR). Bachiller en la Enseñanza del Castellano y Literatura de la Universidad de Costa Rica (UCR). Ha sido profesora de la Enseñanza del Español en el Programa de Educación Abierta del Ministerio de Educación Pública (MEP), en el Colegio Nuevo Mundo (centro educativo privado). Actualmente se desempeña como profesora en el Sistema Educativo Saint Clare (centro educativo privado).

2 Licenciada en Ciencias de la Educación con énfasis en Administración de la Educación de la Universidad de Costa Rica (UCR). Bachiller en la Enseñanza del Castellano y Literatura de la Universidad de Costa Rica (UCR). Ha laborado como profesora en el Colegio Nuevo Mundo, el Colegio Universitario Boston, además de clases particulares a estudiantes; también ha trabajado en el Instituto de Servicios Educativos (ISESA, Sede San Ramón) y como asistente de profesores en la Universidad de Costa Rica, Sede Occidente.
} 
paz", el cual tiene por finalidad ser una alternativa de innovación y cambio, en el que se genere un espacio de convivencia entre la comunidad y la institución; así como fomentar la vivencia de valores entre los integrantes de la comunidad.

Palabras clave. Gestión educativa, liderazgo, innovación, cambio, ambientes complejos.

Abstract. This article discusses the comparative analysis of educational management as an agent of change and innovation to the complex educational environments in organizations: Nuevo Mundo High School and Piedades Sur's Technical High School, San Ramon. To obtain the data we applied a questionnaire to a sample of teachers and students as well as the directors of both institutions. Besides we used multiple secondary sources to build a context of interpretation that would locate the particular case of these two schools, within the reality of educational management as an agent of change and innovation. The principle guiding this study is that it is now essential to implement techniques and strategies, where it is required active leaders with human guidance and clarity in educational management that must be addressed not only in the present but also in the future.

Finally, with the results and conclusions obtained through the analysis of the data, we performed a project proposal called "Week of integration: promoting a culture of peace", which purpose is to be an alternative of innovation and change, which generates a space of coexistence between the community and the institution, as well as promote their experience of values among community members.

Keywords. Educational management, leadership, innovation, change, complex environments.

\section{Introducción}

El gran reto de las organizaciones modernas es animarse a actuar a través del cambio y la innovación, lo cual va de la mano a una mejora institucional con miras a la calidad educativa. Las instituciones que se cierran al cambio no avanzan en este mundo globalizado en que está inmersa la sociedad actual. Por lo tanto, todo el personal directivo debe propiciar herramientas básicas para generar aprendizajes significativos, motivación y calidad educativa.

Con el paso del tiempo se ha ido desarrollando una gran diversidad de acontecimientos promotores de ambientes complejos que repercuten en el sector educativo, por lo que las organizaciones deben hacer frente a todos estos retos existentes para lograr, en la medida de lo posible, mejorar el funcionamiento de las organizaciones y asegurar una enseñanza de calidad, la cual sería capaz de hacer frente a esta sociedad actualmente compleja.

En los tiempos actuales, cualquier tipo de organización y en especial la educativa debe ser competitiva, ya que los cambios, la globalización y los retos emergen con gran intensidad. De esta forma se requiere una gestión activa, promotora del cambio y la innovación ante los complejos ambientes educativos, la cual necesita coordinar y por ende actuar en conjunto con la comunidad educativa.

La gestión educativa como agente de cambio no puede verse como un concepto aislado o responsabilidad única y exclusivamente del director o de la directora de la institución, sino que

\begin{tabular}{l|lr}
\hline 44 & Número publicado el 1 de julio del 2013 & María Alejandra Barquero González \\
Mirieth Montero Matamoros
\end{tabular}


para que sea eficiente y eficaz debe haber una estrecha relación y una efectiva comunicación entre la administración y la organización en general.

El actual contexto educativo es testigo de ambientes complejos, es decir, de la aparición de circunstancias que hacen más difícil el desempeño de la labor administrativa y docente a nivel institucional, sin embargo, estos retos son parte de la tarea directiva. Lograr una gestión institucional exitosa y eficaz es uno de los desafíos más preocupantes que deben enfrentar las organizaciones y el personal directivo.

Los líderes de un centro educativo deben de diagnosticar y analizar a profundidad todo lo que acontezca en la organización educativa y su contexto, puesto que solo de esta manera lograrán, con la ayuda de los demás miembros de la comunidad educativa, crear un plan para propiciar el cambio en el quehacer del centro, que implemente metodologías administrativas y docentes innovadoras. De ahí, la importancia de los objetivos propuestos para este estudio.

\section{Objetivos Generales.}

Realizar un análisis comparativo de la gestión educativa como agente de cambio e innovación ante los complejos ambientes educativos en la práctica organizacional de las organizaciones: Colegio Nuevo Mundo y Colegio Técnico Profesional Piedades Sur de San Ramón.

Diseñar con base en los resultados del análisis comparativo, estrategias de cambio e innovación que permitan un mejoramiento de la gestión educativa para enfrentar y aprovechar los complejos ambientes educativos.

\section{Objetivos Específicos.}

- Describir las funciones teóricas y prácticas de la gestión educativa como agente de cambio e innovación ante los complejos ambientes educativos.

- Identificar los complejos ambientes educativos a los que se enfrenta la gestión de la educación para reconocer la forma en que son abordados.

- Determinar las actividades que permiten a la gestión educativa aprovechar los complejos ambientes educativos para desarrollar cambios e innovaciones.

- Proponer estrategias de cambio e innovación que contribuyan a fortalecer la gestión educativa en las organizaciones ante los complejos ambientes educativos. 


\section{Referencial teórico de la investigación}

Es importante recordar que el personal directivo desempeña un papel fundamental dentro de la organización, pues es el gestor, supervisor, organizador, ejecutor y evaluador del importante proceso de la enseñanza y el aprendizaje, en donde se debe promover la innovación y el cambio, herramienta indispensable para alcanzar el éxito a nivel educativo. Al director o directora le corresponde velar por que se cumpla con los planes y programas, así como permitir la capacitación y actualización del personal docente. Además de evaluar y posibilitar el acceso de los recursos didácticos.

La labor que realiza el personal directivo de una institución es trascendental, pues de este depende el buen funcionamiento de la misma. Si bien es cierto que solo, este personal, no puede llevar a cabo los planes propuestos, es quien tiene la obligación de alentar a su equipo de trabajo para que entre todos logren lo que se tiene planeado. Los miembros de una organización enfocada en la educación, deben tener claro que al lograr los cambios, estarán aportando a la formación integral y al desarrollo de procesos innovadores requeridos para afrontar los retos que imponen los complejos ambientes educativos.

\section{Gestión educativa.}

La gestión es amplia e involucra una gran variedad de componentes. Una de sus funciones tiene que ver con la forma en cómo se administra una organización educativa para el logro exitoso de los objetivos propuestos. Por lo tanto, en la institución educativa, el personal directivo es la máxima autoridad y el encargado de conducir la organización escolar. De tal manera, el profesional en gestión de una institución educativa debe velar por dos dimensiones: la organizacional y la pedagógica. Según García, Rojas y Campos (2002), la dimensión organizativa apunta a las siguientes tareas:

1. Definir el proyecto institucional.

2. Fijación de metas de cambio.

3. Planificación de estrategias.

Con respecto a la dimensión pedagógica, se toman las siguientes acciones como algunas de las tareas de esta área:

1. Trabajar por la cultura y la identidad de la escuela.

2. Explicar las concepciones acerca del aprendizaje.

3. Explicitar los criterios para hacer programas didácticos. (pp. 21-22) 
Aunado a lo anterior, Arroyo (2010) afirma que, en relación con la función del gestor o gestora de la educación:

La teoría no le brinda al director (...), las fórmulas para resolver los problemas o alcanzar los retos de la organización educativa, tan solo constituye el punto de partida, para que mentalmente los examine y establezca las posibles relaciones. Para luego trasladarlas a la acción, mediante el diseño de estrategias, en donde las aptitudes y las habilidades personales del director, contribuyen de manera notable al éxito de la organización. ( $p$. 102)

Asimismo, sobre las diversas tareas que debe desempeñar un profesional en gestión de la educación, Arroyo (2010) menciona que:

Este tiene a su cargo la dirección, coordinación y supervisión de las actividades técnicas y administrativas que se realizan en un centro educativo de segunda enseñanza. Además, señala que el gestor es quien planea, dirige, coordina y supervisa las actividades técnicas y administrativas de la institución a su cargo. También, asesora y orienta al personal en aspectos técnicos y administrativos. Coordina los diferentes programas del centro educativo y vela por su correcta ejecución.

Organiza y dirige actividades administrativas, culturales, cívicas y sociales. Vela por el mantenimiento y conservación del plantel educativo y por el buen aprovechamiento de los materiales, útiles y equipo de trabajo. Promueve la proyección del centro educativo a la comunidad. Coordina y evalúa los resultados de los programas bajo su responsabilidad y recomienda cambios o ajustes necesarios para el logro de los objetivos. (pp. 29-30)

Con base en la información que expone el autor, se demuestra la gran versatilidad que debe tener todo profesional en gestión de la educación, puesto que tiene a su cargo el buen funcionamiento del centro educativo que lidera. Es por esto que, un gestor o gestora no debe fungir como un ente pasivo, sino más bien uno dinámico que genere cambio, transformaciones e innovaciones que propicien un aprendizaje significativo y por ende, una mayor calidad educativa.

El profesional en gestión de la educación de una organización educativa, debe aprovechar sus conocimientos teóricos para aplicarlos en situaciones de conflicto, pero de ninguna manera apagarse a ellos; por el contrario, tiene la obligación de evolucionar, emplear su inventiva y garantizar los procesos de cambio a través de estrategias que propicien un centro educativo de calidad. Por esta razón, es indispensable visualizar a la gestión educativa como un agente de cambio e innovación y por ende, dejar de lado la forma tradicional. 


\section{Liderazgo.}

El profesional en gestión de la educación regula y administra el buen desempeño de la organización; para ello, se hace indispensable que sea una persona capaz de propiciar el cambio y la innovación con actitud firme y convincente. Por esta razón, es fundamental una actitud de liderazgo. Con respecto a este término, Bolívar (2010) menciona que “... entendemos por "liderazgo", fundamentalmente, la capacidad de ejercer influencia sobre otras personas" (p 13).

Es cierto que el personal directivo tiene por sí mismo un papel de líder, pues es el representante del centro educativo, sobre quien cae la mayor responsabilidad respecto al buen o mal funcionamiento del mismo. Además, debe tomar decisiones, guiar y orientar el camino al éxito. Al respecto, Bolívar (2010) afirma que "la capacidad para mejorar de un centro escolar depende, de manera relevante, de equipos directivos con liderazgo que contribuyan a dinamizar, apoyar y animar que aprenda a desarrollarse, contribuyendo a construir la capacidad interna de mejora" (p 11).

El gestor o gestora de la educación debe dirigir con liderazgo su organización educativa, ya que por el contrario, surgiría un caos, una desorganización y un mal desempeño del quehacer educativo. Tiene en su poder el éxito o fracaso de la organización y para ello, se hace indispensable ser un buen líder. Bolívar (2010) asegura lo siguiente: “... ejercer un liderazgo supone ir más lejos induciendo al grupo a trabajar en determinadas metas" (p. 13).

El liderazgo moderno, lleva a una mejor incorporación de habilidades que permitan a los estudiantes y personal docente a un desarrollo más amplio, que les sea útil en la motivación. De ahí, que Bolívar (2010) mencione que:

(....)se están demandando organizaciones escolares más flexibles, capaces de adaptarse a contextos sociales complejos. Las organizaciones con futuro son aquellas que tengan la habilidad para aprender a desarrollarse y hacer frente al cambio. Para lograrlo precisan, entre otras, de autonomía que les posibiliten poner en marcha proyectos propios y aprender de la experiencia. (p. 12)

De esta manera, es importante reconocer que el líder debe ser consciente de su autonomía dentro del centro educativo y con ella, permitir a los docentes y estudiantes innovar para obtener un aprendizaje significativo, a pesar de los ambientes complejos que posea el centro.

\section{Cambio e innovación}

Para que haya un verdadero cambio organizacional se hace necesario el trabajo en equipo que propicie la mejora, el cambio o la innovación dentro de la organización. Al respecto, el Consejo Nacional de Rectores (CONARE, 2006) afirma: 
Los cambios en educación implican cambios en las personas, sea en los administradores, en los educadores y las educadoras, en los y las estudiantes o en los padres de familia. (...) Requieren, para su aceptación, de una participación activa de los involucrados y de un proceso gradual que produzca los cambios esperados. (...) El grado de identificación de los y las participantes con la propuesta de cambio puede asegurar el éxito o fracaso de la propuesta. (p.101)

Lo que difiere un centro educativo de calidad de otro que no lo sea, es su capacidad para sobresalir en la adversidad y buscar, en conjunto, estrategias que propicien la mejora institucional.

En la actualidad, la educación pasa por una etapa en la que es indispensable renovar sus técnicas, ya que con los avances tecnológicos se facilita acceder a la información. Por esto, el proceso de enseñanza y aprendizaje no puede seguir apegado a sus viejos métodos didácticos, más bien debe guiar a los alumnos para utilizar de la mejor manera todos los recursos que tienen a su alcance, esto se logra innovando, concepto que es muy importante manejar para efectos de este trabajo.

(...) Los diversos significados que se atribuyen a las innovaciones están directamente relacionados con la idea de cambio, ya sea de conducta, actitudes, aproximaciones, estrategias o incluso formas de pensamiento. Consideramos relevante en el estudio de la gestión educativa explicitar que este término del cambio, se asocia de manera directa con la implementación y requiere la ubicación del sentido o finalidad de para qué es el cambio, qué es y cómo funciona. (Gómez, 2002, p.5)

Adicionalmente, Venegas (1995) plantea lo siguiente:

Se concibe la innovación educativa como optimización de procesos de mejora intencionales, circunscritos a marcos más específicos, tales como la zona, centro y aula, cuyo núcleo de acción es el centro educativo como institución generadora de cultura. (p.11)

Al tomar en cuenta lo anterior, se evidencia que la innovación lleva implícita la idea de cambio, para que la misma sea efectiva requiere de múltiples factores entre los que destacan el papel de los directivos y docentes quienes deben velar por el mejoramiento de la organización educativa. Según Gómez (2002) los docentes y administrativos son quienes deben tomar las decisiones que tengan que ver con innovación, ellos están llamados a dar el primer paso, teniendo presente que se desea con esto lo mejor para el centro educativo y los estudiantes. Con respecto al tema, Venegas (1995) menciona que: 
El cambio educativo debe estar íntimamente ligado a la calidad de la educación, para que tenga sentido. No se trata únicamente del cambio por el cambio, del cambio por el interés de mostrar algo diferente. El cambio debe tener como último punto de referencia el mejoramiento de la calidad de la educación y que sea así entendido por los docentes, directores, padres de familia, estudiantes y demás personal.

Se debe tratar de que el sistema educativo llegue a ocurrir cambios importantes y valiosos. (pp. 27-28)

Se debe comprender la importancia de la creatividad y el conocimiento como competencias fundamentales para la gestión de organizaciones educativas innovadoras. En la actualidad, uno de los elementos que distingue a una organización de otra es la capacidad individual y organizativa de propiciar el cambio a pesar de los obstáculos, limitaciones o ambientes complejos. Garbanzo y Orozco (2007) mencionan que "... la administración de la educación eficaz y eficiente es el medio oportuno para posibilitar los cambios requeridos en materia educativa" (p. 99). De este modo, se puede afirmar que sobre el personal directivo recae la responsabilidad de impulsar, propiciar y liderar los cambios que garanticen una mejora organizacional.

\section{Complejos ambientes educativos.}

Las organizaciones educativas de hoy deben enfrentar una gran diversidad de desafíos. Los cambios sociales (costumbres, tradiciones...) afectan el comportamiento y aprendizaje de los niños y jóvenes, pues en la actualidad existe un alto riesgo de violencia, desintegración familiar, exclusión social, discriminación, entre otros, que hacen más complejo el ambiente educativo. Al respecto, Garbanzo y Orozco (2007) afirman que:

(...) los variados entornos sociales a los que se somete la educación; entornos característicos por un sinfín de dificultades, como la deserción, la pobreza, la inequidad, la infraestructura, y la preocupante exclusión educativa, entre otros (...) exponen a la administración de la educación a su principal reto: a su capacidad de gestión en aras de la construcción de un desarrollo sostenible con equidad, calidad y justicia. (p. 99)

En relación con la misma temática, Ander-Egg (2004) indica que "la educación tiene ante sí retos de diferente naturaleza: unos como consecuencia de los avances científicos y tecnológicos, algunos derivados de los cambios culturales y modos de vida, otros frutos de las transformaciones socioeconómicas" (p 19).

Ben (2005) indica que uno de los principales obstáculos que deben superar las instituciones educativas es la parálisis que generan las situaciones de conflicto que se originan en ella. Por otra parte, Garbanzo y Orozco (2007) mencionan lo siguiente: 
Para enfrentar los diferentes desafíos que interpelan a la educación, se requiere de un nuevo paradigma de la administración de la educación, epistemológicamente renovada, de manera que el sistema educativo trabaje de acuerdo con parámetros de calidad, como condición para aspirar a lograr, con mayor pertinencia, los fines y objetivos de la educación. (p. 97)

En muchas ocasiones, los centros de enseñanza se ven envueltos en estos ambientes complejos que impiden la sana convivencia e imposibilitan la labor formativa. Sin embargo, asumir una clara interpretación de los retos o desafíos es una condición indispensable para el personal directivo y docente.

La respuesta a todos estos desafíos parece representar una utopía para las organizaciones educativas, pero es posible minimizar la complejidad existente en la medida en que sean abordados, en forma oportuna y responsable. Ander-Egg (2004) afirma que "vivimos en un mundo de complejidad creciente (...), el desarrollo de la ciencia moderna, nos pone de relieve la complejidad de todo lo existente y, de manera particular, de los seres vivos" (p. 125).

Ante un panorama contextual ampliamente complejo, la organización educativa debe asumir con responsabilidad la administración de todos los procesos educativos en procura de resultados educativos de excelencia y calidad. Garbanzo y Orozco (2007) señalan que "un centro educativo bien gestionado, a pesar de la adversidad que lo pueda acompañar, es capaz de producir cambios significativos en el proceso de aprendizaje" (p. 99). Sin embargo, para llevar a cabo estos procesos, en un ambiente de cambio e innovación, es indispensable implementar estrategias que actúen como guías de la transformación educativa.

\section{Referente metodológico}

La investigación es de carácter descriptivo, pues especificó las necesidades de implementar cambios e innovaciones en las organizaciones educativas actuales. Hernández, Fernández y Baptista (2006) indican que la investigación descriptiva "busca especificar las propiedades, las características y los perfiles de personas, grupos, comunidades o cualquier otro fenómeno que sea sometido a análisis" (p. 102).

El enfoque se inclina hacia lo cuantitativo, puesto que pretende analizar, conocer y describir la forma en que la gestión educativa funciona como agente de cambio e innovación ante los complejos ambiente educativos, esto comparando la gestión educativa de los centros educativos: Colegio Nuevo Mundo y Colegio Técnico Profesional Piedades Sur. Al respecto, Fernández y Pértigas (2002) señalan lo siguiente:

La investigación cuantitativa trata de determinar la fuerza de asociación o correlación entre variables, la generalización y objetivación de los resultados a través de una muestra 
para hacer inferencia a una población de la cual toda muestra procede. (p.1)

De tal manera, se presenta un análisis en el desarrollo de las innovaciones en los distintos centros educativos que gestionan desde la administración de la educación, la manera como estas innovaciones ayudan a que el proceso de enseñanza y aprendizaje se dé en buenas condiciones ante los complejos ambientes educativos que se ven en la sociedad.

El tipo de muestra de la investigación es no probabilística. De acuerdo con Hernández et al. (2006) "en las muestras de este tipo, la elección de los sujetos no depende de que todos los sujetos tengan la misma probabilidad de ser elegidos, sino de la decisión de un investigador o grupo de encuestadores" (p.167). La muestra no probabilística posee varias clases, en esta investigación se pretende utilizar la muestra de sujetos voluntarios, la cual Hernández et al. (2006) la definen como: "el investigador elabora conclusiones sobre especímenes que llegan a sus manos de forma casual" (p.168). En este tipo de muestra se estaría hablando de los docentes, una muestra de la población estudiantil (específicamente los estudiantes presidentes de sección) y los directores de ambas instituciones.

Según los resultados que se obtengan de las muestras seleccionadas, de cada uno de los colegios, es que se podrá realizar una comparación sobre las técnicas que se utilizan en uno que se pueden tomar en cuenta y ser utilizadas en otro, puesto que los resultados al ser analizados, determinarán la forma más adecuada para innovar.

Como técnicas e instrumentos se utilizó un cuestionario y una entrevista (aplicada a los directores), diseñadas con base en la información pertinente del tema en estudio para alcanzar los objetivos propuestos.

Para la validación se realizó un plan piloto de los instrumentos por aplicar. Varios docentes, estudiantes y un director que no eran parte del estudio, revisaron el documento, dieron sus opiniones y sugerencias para lograr la mayor transparencia y comprensión de cada una de las interrogantes, pues de esta manera se recogerían los datos pertinentes para la investigación.

Los sujetos que se considerados como informantes claves de esta investigación son los directores (as) de cada una de las organizaciones educativas; además, de una muestra no probabilística de docentes y estudiantes de cada institución.

La cantidad total de docentes encuestados de los Colegios Nuevo Mundo fueron 11 y Colegio Técnico Profesional de Piedades Sur, diez. En total, los docentes encuestados fueron 21, esto con efecto de comparar los porcentajes de los números absolutos. Todos los docentes son regulares dentro de la institución. En cuanto a la muestra de estudiantes, se pudo encuestar a 10 jóvenes del Colegio Nuevo Mundo y a 17 del Colegio Técnico Profesional de Piedades Sur de San Ramón. Asimismo, se logró obtener datos muy valiosos por parte de los directores de ambas instituciones educativas. 


\section{Análisis de la información}

A continuación, se muestra la sistematización y análisis de los datos obtenidos en el Colegio Técnico Profesional de Piedades Sur de San Ramón y en el Colegio Nuevo Mundo, en relación con la opinión del personal docente y de la población estudiantil, en torno a los objetivos del estudio.

\section{Datos suministrados por el personal docente en relación con la gestión educativa como agente de cambio e innovación en ambientes educativos complejos.}

Las funciones administrativas en las organizaciones educativas son esenciales para lograr el cambio y la innovación requeridos en un ambiente social complejo. La dinámica social afecta, directamente, el quehacer del personal directivo, el cual debe actualizarse para afrontar los desafíos de un entorno complejo. La principal herramienta ante los constantes cambios en las formas de aprender, de actuar y enseñar exigen innovaciones en los estilos administrativos y esencialmente en su función directiva.

El personal docente considera que la gestión de una determinada institución educativa se basa en la buena organización de la misma. Desde el punto de vista del personal directivo quien, junto con sus colaboradores determina las pautas a seguir, en lo que se refiere a la planificación y coordinación del personal, la población estudiantil, madres y padres de familia, para el logro de todos los objetivos planeados, conjuntamente. Es importante que el personal directivo posea un mayor contacto comunicacional con su personal de trabajo y con la población estudiantil, capacidad para delegar funciones y ejercer un liderazgo participativo permanente, para un apropiado y oportuno desarrollo del proceso de enseñanza y aprendizaje.

Según la UNESCO (2000):

La gestión educativa puede entenderse como las acciones desarrolladas por los gestores que pilotean amplios espacios organizacionales. Es un saber de síntesis capaz de ligar conocimiento y acción, ética y eficacia, política y administración en procesos que tienden al mejoramiento continuo de las prácticas educativas; a la exploración y explotación de todas las posibilidades; y a la innovación permanente como proceso sistemático. (p. 16)

Es este sentido, el personal directivo debe promover en todo momento por el desarrollo óptimo del centro educativo que tienen a cargo y la organización del personal colaborador del área administrativa, y el personal técnico docente. Esto con la finalidad de lograr una institución educativa de calidad, en todos los ámbitos, el administrativo, técnico y el docente.

El cambio es una constante en las organizaciones y la innovación un imperativo en el personal directivo. La capacidad para lograr este proceso es determinante y es indispensable conocer el impacto en el personal que genera la labor del personal directivo. 
Aunque el personal directivo de un determinado centro educativo cuente con el personal administrativo y docente que participa en la coordinación y dirección de la institución, este es, en última instancia, quien debe estar atento a incorporar todas aquellas metodologías técnicas administrativas y educativas novedosas que generen cambios apropiados a las demandas y problemáticas, de la organización educativa, para la promoción de un proceso de enseñanza y aprendizaje innovador y contextualizado, con significado para el personal docente y la población estudiantil, acorde con los retos y desafíos de un contexto educativo complejo.

Es importante destacar que, en su mayoría, el personal de ambas instituciones educativas opina que es una necesidad y obligación del personal directivo innovar y enfrentar creativamente el cambio. La convergencia del personal docente de ambos centros educativos es que innovar si contribuye a contrarrestar los ambientes complejos, pues son muy necesarios de aplicar en el mundo actual, ya que la globalización y los avances tecnológicos hacen necesario el uso de nuevas metodologías que se adapten a los intereses de la población estudiantil actual.

Aquí se muestra la opinión del personal docente, en relación con la importancia que tienen la innovación y el cambio en la educación actual. Especialmente, las características del entorno social actual y sus exigencias:

- Innovar es una responsabilidad.

- Innovar permite libertad para planear y ejercer la docencia.

- Cambiar e innovar permite un mejor desempeño.

- Innovar con base en las debilidades y fortalezas de la organización.

- Cambiar e innovar permite un mejor desempeño.

La tendencia a seleccionar una negativa o positiva entre el personal docente ante el cuestionamiento de si el accionar del personal directivo permite implementar la innovación y el cambio, se dividió de la siguiente manera:

De los informantes del Colegio Nuevo Mundo (CNM), el 54\% contestó afirmativamente, por su parte el $46 \%$ opinó que no. En el caso del Colegio Técnico Profesional de Piedades Sur de San Ramón (CTPPS), el 55\% de los profesores opinaron que sí, frente a un $45 \%$ que considera que no. En ambas instituciones educativas las respuestas están divididas, la mayoría se inclina por que sí se permite innovar. Sin embargo, es de gran interés que la otra parte, casi la mitad, opine que no, lo cual indica que se permite el cambio, pero no necesariamente eso significa que este se lleve a cabo. 
Gráficos 1 y 2. Opinión del personal docente en relación con la utilización de técnicas innovadoras en la institución
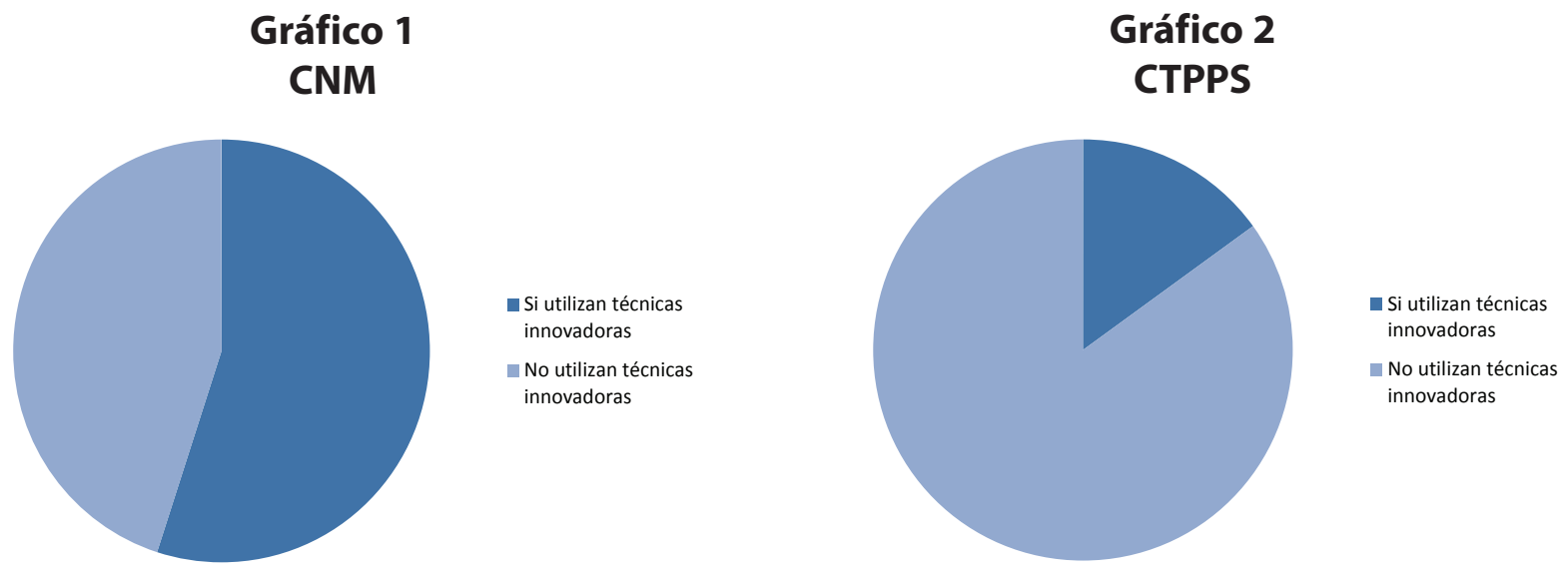

Nota: Cuestionario aplicado a la población docente encuestada del Colegio Nuevo Mundo y del Colegio Técnico Profesional de Piedades Sur de San Ramón.

El personal docente de los colegios CNM y CTPPS opina en relación con la utilización de técnicas innovadoras en la institución, según se aprecia en los gráficos 1 y 2, que los informantes del Colegio Nuevo Mundo contestaron en un $85 \%$ que no y un $15 \%$ contestó que sí. Por su parte, los profesores del Colegio Técnico Profesional de Piedades Sur, contestaron en un $45 \%$ que sí y opinaron que no, el 55\% de los entrevistados.

En relación con el concepto de ambiente educativo complejo en la institución, el personal docente menciona que las drogas, la violencia, la indisciplina y el desinterés de los padres y madres de familia son los principales factores que calificarían como ambientes complejos, al menos son los que ambos colegios citan, mismos que son problemáticas de la sociedad, que se pueden encontrar en distintos lugares del territorio nacional, lo cual se refleja con las instituciones que son objeto de estudio, pues una se encuentra en el centro de San José (CNM), zona urbana, mientras que la otra se ubica en Piedades Sur de San Ramón, zona rural, lo que no impide que los problemas sociales sean los mismos, a pesar de la lejanía y diferencia geográfica, a continuación los y las docentes afirman:

Informante 3: daño a la propiedad privada de vehículos.

Informante 4: indisciplina por parte de algunos estudiantes.

Informante 5: drogadicción (CTPPS). 
Además, se suman otras problemáticas que son específicas de cada institución y que, en resumen, permiten ver que ambientes complejos para los docentes son todos los factores que limitan o intervienen en el buen desempeño del estudiante en la institución, con su aprendizaje específicamente. Un problema es la rotación de ingreso estudiantil, en diferentes momentos del ciclo lectivo. Según lo manifiesta el Informante 5:"constante ingreso de estudiantes en diferentes periodos del año lectivo". Esto implica, a nivel de los procesos de enseñanza y aprendizaje, recurrir a las adecuaciones necesarias con los estudiantes de nuevo ingreso y provoca entonces, un mayor esfuerzo del personal docente para cumplir con el programa de estudios y poner a nivel requerido a todo el grupo estudiantil.

\section{Datos suministrados por la población estudiantil en relación con la gestión educativa como agente de cambio e innovación en ambientes educativos complejos.}

Los estudiantes de ambos colegios visualizan la figura del personal directivo como un ente encargado de dirigir con eficacia la parte académica y administrativa del centro educativo, con el fin de mantenerlo en pie.

La mayoría de los encuestados opina que es importante que, en la institución, se propicie el cambio y la innovación, con el fin de lograr mejores resultados, motivación y satisfacción en la comunidad educativa. Por ejemplo, el informante 4 del Colegio Nuevo Mundo opina que, al impulsar el cambio y la innovación, se "garantiza la promoción de los estudiantes a un mejor futuro dándole al estudiante un mejor lugar para su aprendizaje". El informante 7 del Colegio Técnico Profesional de Piedades Sur opina que la innovación contribuiría "para que los estudiantes tengas más ganas de estudiar y hacer las cosas".

Es importante rescatar que los estudiantes de ambos colegios ven la innovación como un medio para el desarrollo de capacidades, estrategias, retos y metas que servirán de herramienta para desenvolverse efectivamente en sociedad.

Estos estudiantes manifiestan, a través de sus opiniones, que la innovación y el cambio son una oportunidad para generar aprendizaje más significativo y duradero. Es decir, aprender por medio de su propia experiencia, pero para ello se requiere de una transformación de las ideas y prácticas educativas tradicionales. Además, de proyectos y actividades lúdicas que generen interés y compromiso. 
Tabla 1.

Opinión de la población estudiantil del CNM y CTPPS en relación con su participación desde la

\begin{tabular}{|c|c|c|c|}
\hline CNM & $\begin{array}{l}\text { CTP PIEDADES SUR DE SAN } \\
\text { RAMÓN }\end{array}$ & DIFERENCIAS & SIMILITUDES \\
\hline $\begin{array}{l}\text { INF 2: cuando tenemos } \\
\text { algún problema se nos } \\
\text { atiende. } \\
\text { INF 4: tribunal y gobierno } \\
\text { estudiantil. } \\
\text { INF 6: nos toman en } \\
\text { cuenta para actividades. } \\
\text { INF 8: se preocupan por } \\
\text { nuestras opiniones y nos } \\
\text { toman en cuenta en los } \\
\text { cambios que realiza el } \\
\text { colegio. } \\
\text { INF 10: para mejoras. }\end{array}$ & $\begin{array}{l}\text { INF 8: En realidad solo } \\
\text { algunos. } \\
\text { INF 11: A través de } \\
\text { propuestas al director } \\
\text { y recolección de firmas } \\
\text { para impulsarle. }\end{array}$ & $\begin{array}{l}\text { Los estudiantes del } \\
\text { CNM manifiestan } \\
\text { que en ocasiones } \\
\text { son tomados en } \\
\text { cuenta, mientras } \\
\text { que los del CTP no. }\end{array}$ & $\begin{array}{l}\text { Ambas } \\
\text { poblaciones } \\
\text { educativas } \\
\text { solicitan ser } \\
\text { tomados en } \\
\text { cuenta para } \\
\text { propiciar el } \\
\text { cambio y la } \\
\text { innovación. }\end{array}$ \\
\hline
\end{tabular}

Dirección para propiciar el cambio y la innovación, 2011.

Nota: Cuestionario aplicado a la población estudiantil encuestada del CNM y CTP Piedades Sur de San Ramón.

A los estudiantes que respondieron ser tomados en cuenta por el personal directivo para propiciar el cambio y la innovación, se les solicitó que indicaran de qué forma eran considerados en la labor educativa. El informante 2 del Colegio Nuevo Mundo señala que son tomados en cuenta cuando tienen algún problema, ya que son atendidos de inmediato. También, el informante 8 del CNM indica que se preocupan por sus opiniones y los toman en cuenta en los cambios que realiza el colegio. Con respecto al Colegio Técnico Profesional Piedades Sur, el informante 11 señala que son tomados en cuenta a través de las propuestas al director y la recolección de firmas para impulsarle.

En relación con la definición que da la población estudiantil de lo que debe ser un profesional en gestión educativa, el informante 4 del CNM señala que debe ser una persona segura, que interactúe con los estudiantes. El informante 5 menciona que necesita ser una persona creativa, novedosa, dinámica y flexible. El informante 8 cree que debe ser alguien que se preocupe por los estudiantes y por formar personas de calidad estudiantil. 
Las opiniones de los estudiantes encuestados en el CTPPS son muy similares a las del CNM; por ejemplo, el informante 1 piensa que el director debe relacionarse más con los estudiantes y no pasar tanto tiempo en la dirección, lo cual concuerda con el informante 4 del CNM. También los informantes 8 y 17 del CTPPS consideran que debe ser una persona que se preocupe por los estudiantes y por la institución, lo cual concuerda con la opinión del informante 8 del Colegio Nuevo Mundo.

Con respecto a lo que es una técnica innovadora, el informante 1 del CNM opina que son actividades nuevas que animan a los estudiantes a interesarse por la materia. El informante 7 dice que es utilizar nuevas tecnologías para el rendimiento académico. El Informante 8 menciona que son propuestas nuevas que ayudan a la institución que beneficien al personal docente y a los estudiantes.

Algunas de las opiniones de los estudiantes del CTPPS son como la del informante 1, que indica que una técnica innovadora es tratar de hacer cosas que no se hayan hecho para salir de la rutina. El informante 7 menciona hacer cosas dinámicas y con imaginación y creatividad. El informante 11 indica que son los métodos nuevos de educar a los estudiantes, para un mejor aprendizaje.

A través de lo que manifiestan los estudiantes por medio de la encuesta, se puede afirmar que la mayoría se encuentra con deseos de experimentar cambios e innovaciones en su centro educativo. Los encuestados expresan que, en escasas ocasiones, se realizan actividades o proyectos de carácter innovador en sus colegios, pero que desearían que eso se efectuara con mucha más frecuencia.

Refiriendo la importancia de la innovación en la dirección del centro educativo actual, los encuestados creen que la innovación es importante porque motiva a los estudiantes a seguir estudiando. Por ejemplo, el informante 1 del CNM opina que si hubiera innovación habría estudiantes más activos y comprometidos con el estudio. El informante 6 piensa que la innovación incentiva al estudiante a aprender, dejando a un lado lo tradicional.

Los informantes del CTPPS opinan muy similar a los del CNM. El informante 7 piensa que innovar desde la dirección incentiva a los estudiantes a tener más ánimos y ganas de estudiar. También el informante 10 afirma que la dirección es la cabeza de la institución y si esta innova, se innova todo.

\section{Conclusiones}

Los estudiantes de ambos colegios (Colegio Nuevo Mundo y Colegio Técnico Profesional de Piedades Sur) están de acuerdo en que es el personal directivo quien debe velar para que la 
innovación sea una constante dentro del centro educativo, esto para que se alcance un mayor rendimiento en las funciones de cada miembro del sistema educativo (gestores, docentes, estudiantes, padres de familia), ya que la educación debe mantenerse a la vanguardia con los cambios que experimenta la sociedad en la que se encuentra inmerso el centro educativo.

Se comprobó que, a pesar de los esfuerzos de la gestión y de algunos docentes de ambas organizaciones educativas, las técnicas innovadoras que se tratan de implementar no son del todo satisfactorias para los estudiantes. Los estudiantes esperan que los docentes sean más constantes en la implementación de las nuevas técnicas, no que sea algo esporádico, además, que las actividades innovadoras contribuyan directamente a enriquecer la creatividad y buenas ideas de los estudiantes

Ligada a la conclusión anterior, se destaca el hecho de que los estudiantes no consideran ser tomados en cuenta para la implementación del cambio e innovación en los centros educativos CNM y CTPPS.

Para los estudiantes de los colegio CNM y CTPPS, un buen gestor es aquel que es activo y busca innovar, quien los escucha y los toma en cuenta. Un profesional capaz de salir de las cuatro paredes de su oficina y se acerca más a los estudiantes, los escucha y trata a toda costa de suplir sus necesidades, que sea flexible sin perder su autoridad, ya que ellos necesitan que los orienten y corrijan, con razones justificadas y que posean la intención de formarlos como individuos integrales y no solo por hacer cumplir reglas.

Los estudiantes entienden por técnicas innovadoras, todas aquellas actividades que se salen de la rutina, todo lo que lleve implícita la creatividad y permita a los estudiantes crear conocimiento nuevo para el beneficio del colegio y de la comunidad estudiantil; lo que para ellos representa una parte muy importante que les facilite el aprendizaje, en el sentido de que sean métodos y técnicas que refuerce los conocimientos previos y que fije los nuevos, a través de la práctica, experimentación, análisis críticos.

Otra conclusión importante para resaltar, es el hecho de que los estudiantes de ambos colegios opinan que, en sus respectivos centros educativos, no se emplean técnicas innovadoras, de acuerdo con lo que ya se determinó que es para ellos innovar.

En ambos centros educativos hay ambientes complejos. La violencia, la repitencia, la indisciplina y los vicios son los principales factores o evidencia de ambientes complejos. Esto es de vital importancia, ya que ellos son los directamente afectados por estos factores y si ellos los determinan complejidad, se les debe prestar atención y soluciones.

En los dos colegios (CNM y CTPPS) que se llevó a cabo la encuesta, los estudiantes opinaron que sí se puede aprovechar los ambientes complejos para propiciar un cambio dentro de la 
institución y fuera de ella. Principalmente, siendo conscientes de lo que está mal para corregirlo: el informante 5 del CNM menciona: "aprendiendo de los errores y realizando cambios que mejoren".

Se concluye además, que las zonas en las que se encuentran ubicados los colegios son consideradas por los mismos estudiantes como inseguras, lo cual también viene a afectar al centro educativo.

Los padres de los estudiantes no demuestran un total interés por el estudio de sus hijos e hijas, dejan la mayor parte de la responsabilidad en el centro educativo, al cual llegan si acaso al final del ciclo lectivo, si sus hijos o hijas no deben presentar o no lograron pasar de nivel.

Los docentes del CNM y CTPPS opinan que la dirección de los colegios es el ente encargado de organizar, planificar, dirigir por buen camino la organización educativa, esto con el propósito de que la educación de cada uno de los estudiantes sea más significativa.

El profesional en gestión de la educación debe ser un ente unificador que está al pendiente de las necesidades de sus colaboradores y estudiantes, lo que permita el diálogo y la constante interacción con todos los individuos que conforman la organización educativa (docentes, estudiantes, padres, madres y encargados).

Los docentes concluyen que sí es importante innovar y hacer cambios en las organizaciones educativas, ya que es indispensable que las organizaciones educativas estén a la vanguardia con los cambios que sufre la sociedad a raíz de la globalización y avances tecnológicos.

Los docentes aseguran que sí son tomados en cuenta sus aportes para generar innovación y cambio, pero esperan aún más apoyo por parte de la gestión, que la dirección de las respectivas instituciones se salga de las cuatro paredes y puedan colaborar más directamente para que esos avances puedan llevarse a cabo satisfactoriamente.

En cuanto a la participación de los estudiantes en la creación de técnicas innovadoras, los profesores encuestados concluyen que lo jóvenes no se involucran lo suficiente en ese tema. A los estudiantes si les llama la atención este tipo de cambios, pero ellos no buscan proponer técnicas, solo lo que lleve en docente o determinen en la institución.

Otra conclusión importante, es que los docentes de las organizaciones educativas ya mencionadas, insisten en la necesidad de que el profesional en gestión de la educación sea una persona que se comunique y esté pendiente de todo lo que ocurre en el centro educativo tomando la opinión de todos, demostrando sus interés en todo y todos.

Además, según la información suministrada por los docentes, en el CNM y CTPPS, la gestión no emplea la innovación y el cambio, ya que los gestores en la mayoría de ocasiones se enfocan más en el área curricular, con el rendimiento académico propiamente dicho. 
Los docentes de los centros educativos están de acuerdo en la definición de ambientes complejos. Opinan que tienen que ver con todo aquello que interfiera en el óptimo aprendizaje de los estudiantes.

Los docentes concluyen que los ambientes complejos que poseen sus instituciones son la desintegración familiar, la drogadicción, la violencia, que provocan muchas veces la indisciplina de los estudiantes.

Se concluye, además, que los ambientes complejos sí deben ser aprovechados para propiciar el cambio y la innovación, puesto que esa complejidad hace que se busquen nuevas salidas y soluciones. En este caso, resulta evidente que se debe cambiar la forma como se han venido haciendo las cosas para lograr obtener resultados distintos que sean más satisfactorios.

Los docentes aportan como sugerencia que se busque involucra más a los padres, madres y encargados, que se les solicite un mayor compromiso con sus hijos e hijas, para que se pueda realizar un trabajo conjunto que logre dar buenos y mejores resultados.

\section{Proyecto "Semana de integración: fomentando una cultura de paz"}

La propuesta de este proyecto tiene por finalidad, brindarle a las instituciones que sirvieron como objeto de estudio para esta investigación, una alternativa de innovación y cambio, el cual pretende generar un espacio de convivencia entre la comunidad y la institución; así como fomentar la vivencia de valores entre los integrantes de la comunidad educativa.

Este proyecto lleva por nombre "Semana de integración: fomentando una cultura de paz". Se pretende que, en una semana por trimestre, se lleve a cabo el proyecto, el cual consistiría en la presentación de diferentes actividades propuestas por los estudiantes y supervisadas por los docentes, las cuales incluirían la presencia de los padres de familia y personas de la comunidad.

Con este proyecto, se procura valorar la creatividad de los alumnos, fomentar valores como el compañerismo, el trabajo en equipo, la tolerancia; también propiciar una mayor comunicación y compromiso entre los miembros de la comunidad educativa.

La Organización de las Naciones Unidas para la Educación, la Ciencia y la Cultura (UNESCO), realiza una traducción del libro de los Siete saberes necesarios para la educación del futuro, de Morin (1999), en donde se expone lo siguiente:

La comprensión es a la vez medio y fin de la comunicación humana. El planeta necesita comprensiones mutuas en todos los sentidos. Dada la importancia de la educación en la comprensión a todos los niveles educativos y en todas las edades, el desarrollo de la comprensión necesita una reforma planetaria de las mentalidades; esa debe ser la labor de la educación del futuro. (p. 53) 
Con base en el fragmento anterior, se justifica la necesidad de propiciar un espacio de comunicación que genere una efectiva convivencia en las futuras generaciones.

Para la implementación de este proyecto, es indispensable la colaboración, apoyo y orientación del profesional en gestión de la educación, pues este debe ayudar para que se logren con éxito los objetivos propuestos. Al respecto, Venegas (2000) plantea que la dirección del centro educativo debe ser un profesional humilde, que escucha a los demás, con confianza en el ser humano que labora junto a él y que valora realmente a todos los colaboradores del centro educativo, pues admite que todos tienen mucho que aportar.

Se propuso este proyecto de integración porque la mayoría de los estudiantes encuestados manifiestan que en su centro educativo no se realizan actividades innovadoras que los motiven a permanecer identificados con su institución. Además, no solo se lograría la participación, innovación, creatividad y aporte de los estudiantes sino que se crearía un espacio lúdico y de aprendizaje significativo que propiciaría la vivencia de los valores que tanto hacen falta en la sociedad actual y que, de una u otra forma, ayudarían al desarrollo de una sociedad más consciente de la necesidad del trabajo en equipo, el respecto de las ideas del otro, la propuesta de proyectos auténticos y el desarrollo de todo su potencial para poder sacar provecho a los talentos y habilidades.

El hecho deque se ponga en práctica este proyecto, motivaría en gran medida al estudiantado, pues son ellos mismos los que sugieren ser tomados en cuenta por el personal directivo.

La convivencia y el disfrute de actividades sanas traerían muchos beneficios, tanto a la institución como a la sociedad en general, pues si los jóvenes están motivados y entretenidos tendrán menos riesgo de ser seducidos por la vagancia, el conformismo, la drogadicción, alcoholismo, delincuencia, entre otros vicios sociales.

El Ministerio de Educación Pública ha querido impulsar en los centros educativos una propuesta similar a la que se expone en este proyecto. Han propuesto un programa llamado "Convivir", el cual recoge la experiencia desarrollada en gran cantidad de centros educativos que actualmente tienen en marcha actividades que en sí mismas son estrategias de promoción de la convivencia. Por esta razón, es pertinente poner a trabajar proyectos que impulsen el disfrute de actividades provechosas para todos.

\section{Objetivo general}

- Propiciar un espacio de convivencia entre la comunidad y la institución educativa con la finalidad de contrarrestar los ambientes complejos educativos a través del cambio y la innovación. 


\section{Objetivos específicos}

- Valorar los aportes de los estudiantes y docentes a la hora en que la institución se va a proyectar a la comunidad.

- Fomentar la vivencia de los valores en las relaciones humanas.

- Propiciar mayor comunicación y compromiso entre los miembros de la comunidad educativa.

\section{Implementación del proyecto "Semana de integración: fomentando una cultura de paz".}

Con esta propuesta se pretende que los estudiantes se involucren en el proyecto, de hecho serán ellos quienes planificarán, organizarán y ejecutarán las actividades. Por tal razón, es labor del personal directivo y docente procurar la motivación y aplicación de diferentes estrategias metodológicas para que los jóvenes aprecien, disfruten, expresen, vivan y fortalezcan la convivencia, la promoción de los valores y la cultura de paz, tanto en el centro educativo como en la comunidad. Morin (1999) menciona lo siguiente.

(...) educar para la comprensión humana (...) ahí se encuentra justamente la misión espiritual de la educación: enseñar la comprensión entre las personas como condición y garantía de la solidaridad intelectual y moral de la humanidad. (p. 47)

Con esta propuesta se pretende fomentar una cultura de paz, de comunicación y de convivencia entre los diferentes miembros de la comunidad educativa. Por lo tanto, el centro educativo viene a ser el medio que facilita la innovación y el cambio hacia las generaciones futuras.

Este proyecto pretende involucrar a la comunidad, por tal razón, se seleccionará una semana en cada trimestre y se invitará a los padres de familia y vecinos de la comunidad a que sean partícipes del esfuerzo, dedicación y talento de los estudiantes. Es importante destacar que para lograr el resultado esperado los alumnos han tenido que pasar por todo un proceso de planificación, aprender a trabajar en equipo, asumir responsabilidades, disponer de una comunicación asertiva y resolver problemas en forma pacífica. Esto preparará a los jóvenes para enfrentarse al futuro.

A continuación, se expondrán, una a una, las siguientes actividades por realizar: planificar la semana, organizar, ejecutar y evaluar. 


\section{Planificación de la semana}

El proceso de planificación de la semana de integración se llevará a cabo con la ayuda activa de los estudiantes, quienes guiados por sus profesores, determinarán las actividades por realizar de acuerdo con las habilidades y destrezas de los mismos alumnos.

El personal directivo también debe mantener una comunicación continua con el personal docente para establecer las actividades que se llevarán a cabo y las funciones respectivas de los participantes. Tanto el personal docente como el personal directivo, deben estar conscientes de cuáles son los principales ambientes complejos que tiene la institución, así podrán guiar a los estudiantes para que las actividades que realicen generen un cambio en dichas conductas contraproducentes, por ejemplo, si ya se sabe que un ambiente complejo dentro del centro educativo Colegio Técnico de Piedades Sur es la violencia o pleitos entre estudiantes, se deben organizar actividades que fomenten la paz, tales como organizar grupos musicales, campeonatos de fútbol, así los jóvenes centrarán su atención en aquellas actividades que les gusta realizar. De ahí, la importancia de tomar la opinión de los estudiantes para organizar las actividades.

\section{Organización de la semana}

La organización de la semana se debe hacer en conjunto: personal docente, personal directivo y representantes de los estudiantes que están a cargo de la organización y con la presencia de padres de familia, para que todos los que son parte del proceso de enseñanza y aprendizaje estén enterados de la organización y sean parte de la misma.

Las actividades deben proyectarse a la comunidad y hacia la misma institución, de esta forma, se podrán organizar actividades a lo interno del centro educativo, tales como campeonatos de fútbol, básquet, volibol u otros deportes, según sea la preferencia de los mismos estudiantes. En los campeonatos pueden participar los miembros del personal docente, esto para que convivan aún más con los estudiantes y compartan con ellos de una manera más libre.

Para las demás actividades, con las cuales se proyecten hacia la comunidad, se pueden incluir charlas de interés público relacionadas con la misma sociedad. Los temas son muy variados, por tal motivo es importante que sean propuestos por los mismos estudiantes, padres y madres de familia, puesto que hacia ellos principalmente irán dirigidos, ya que son miembros directos de la organización educativa.

En el caso del Colegio Técnico de Piedades Sur, en el que se dan diversas especialidades, dentro del plan pueden incluir pequeños talleres para las personas de la comunidad que los deseen aprovechar, los estudiantes mismos los pueden impartir, ya sea enfocado a adultos mayores o comunidad, en general. 
Una actividad que debe ser bien planeada y organizada es la venta de comidas, esto para financiar proyectos de los mismos estudiantes que contribuyan al mejoramiento de centro educativo en todos los ámbitos, incluso para comprar artículos como instrumentos musicales, deportivos o tecnológicos que sirvan en futuras semanas de integración.

\section{Ejecución de la semana}

Una vez que se tienen claros los procedimientos que se deben llevar a cabo para la ejecución de la semana de integración, es el momento de poner en práctica toda la teoría.

Cada persona que participe deberá tener a la mano el cronograma de las actividades y el planeamiento correspondiente, esto para que sepan los pasos por seguir y se agilicen los procesos.

La ejecución del proyecto no está enfocada solo a la semana que se haya determinado para realizar las diversas actividades, sino que se toman en cuenta las semanas previas en las que las personas responsables de realizar la propaganda y los ensayos para las presentaciones, ya que estas labores son parte de las actividades por realizar. Desde ese momento, el personal directivo juega un papel importante, en trabajo conjunto con los docentes en la supervisión del cumplimiento de las actividades, con él único propósito de asegurar un buen desempeño de los y las estudiantes, para que se logren todos los objetivos planteados.

\section{Evaluación de la semana}

La evaluación del proyecto, al igual que su planificación, se hará con la participación del personal directivo, el personal docente, de los y las estudiantes, los padres de familia y la comunidad en general, en la cual está ubicado el centro educativo y que asistieron a las actividades.

Se debe determinar un instrumento para evaluar las actividades, dentroy fuera de la institución. Dentro de la organización educativa se pueden hacer reuniones con los participantes del proyecto y comentar con el FODA, fuera de la institución podría ser útil un cuestionario que sea aplicado a los familiares y amigos más cercanos de los estudiantes y miembros del centro educativo.

\section{Referencias}

Ander- Egg, E. (2004). Los desafíos de la educación en el siglo XXI. Argentina: Ediciones Homo Sapiens. 
Arroyo, J. (2010). Gestión estratégica de organizaciones. San José, Costa Rica: Editorial UCR.

Ben. A. (2005). Problemáticas de la gestión educativa: la inserción en la escuela de alumnos con dificultades en la convivencia. Revista Iberoamericana de Educación. Recuperado en: http:// www.rieoei.org/deloslectores/1156Ben.pdf

Bolívar, A. (2010). El liderazgo educativo y su papel en la mejora: una revisión actual de sus posibilidades y limitaciones. Revista Psicoperspectivas individuo y sociedad, 9(2), juliodiciembre, pp. 9- 33. Recuperado en: http://www.scielo.cl/pdf/psicop/v9n2/art02.pdf

Consejo Nacional de Rectores (CONARE). (2006). Hacia un modelo educativo para elevar la calidad de la educación costarricense: una propuesta de políticas, estrategias y acciones. Oficina de Planificación de la Educación Superior. San José, Costa Rica: EUNED.

Fernández, S. y Pértegas, D. (2002). Investigación cuantitativa y cualitativa. España: Cad. Aten Primaria.

Garbanzo, G. y Orozco, V, H. (2007). Desafíos del sistema educativo costarricense: Un nuevo paradigma de la administración de la educación. Revista Educación, 31(2), pp. 95-110. Recuperado en: http://www.latindex.ucr.ac.cr/educacion-31-2/06-GARBANZO.pdf

García, N.; Rojas, M. y Campos, N. (2002). La administración escolar para el cambio y el mejoramiento de las instituciones educativas. San José, Costa Rica: Editorial UCR.

Gómez, M. (2002). La innovación y cambio para el mejoramiento escolar. Universidad Pedagógica Nacional. Recuperado en: http://www.lie.upn.mx/docs/DiplomadoPEC/In yMGGM.pdf

Hernández, R.; Fernández, C. y Baptista, P. (2006). Metodología de la investigación. México: McGraw Hill.

Morin, E. (1999). Los siete saberes necesarios para la educación del futuro. París: UNESCO. Recuperado en: http://unesdoc.unesco.org/images/0011/001177/117740so.pdf

Organización de las Naciones Unidas para la Educación, la Ciencia y la Cultura (UNESCO). (2000). Gestión educativa estratégica. Instituto Internacional de Planeamiento para la Educación. 
Ministerio de Educación de la Nación. Recuperado en: http://www.colombiaaprende.edu.co/ html/home/1592/articles-189023_archivo_2.pdf

Venegas, P. (1995). Innovación y cambio en educación. El director y el docente como partícipes medulares. San José, Costa Rica: EUCR.

Venegas, P. (2000). Principios, fuentes y referencias de la administración educativa moderna. San José, Costa Rica: EUCR. 